\title{
fúnel en nueva orleáns
}

WAYNE F. PALMER, Presidente de Palmer and Baker Engs, Inc.

La ciudad de Nueva Orleáns, Luisiana (EE. UU.), tiene un recio sabor antiguo, distinguiéndose de otras ciudades de este país por su propia atracción y un enorme tráfico. El río Mississipí circunda la ciudad constituyendo un gran lago. Si éste ha sido el elemento básico del gran desarrollo que ha logrado este centro urbano, la red de canales que de él se derivan, junto con su propio cauce, han sido la causa de un grave problema de circulación, fluvial y por carretera.

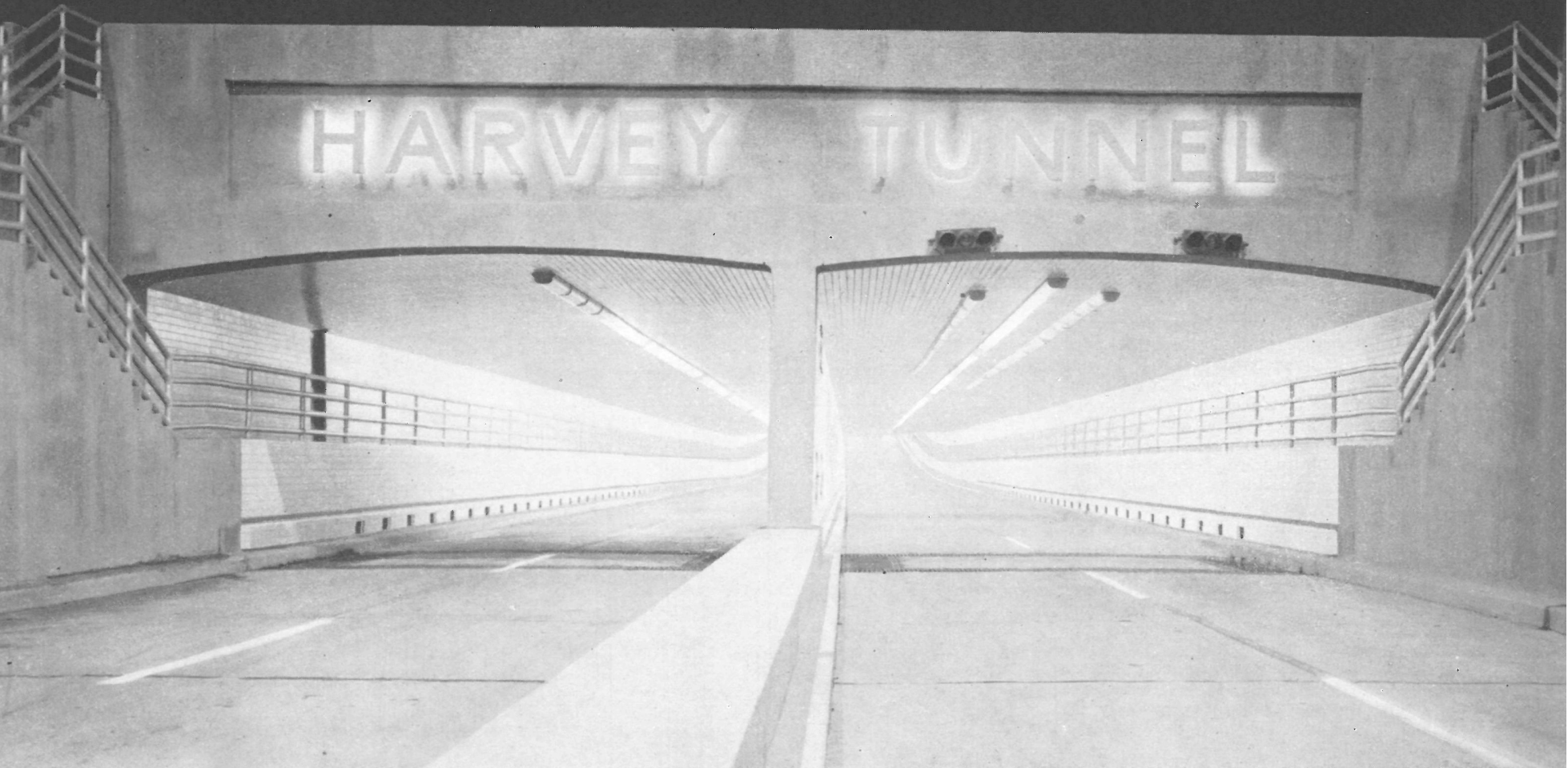

El nudo más acentuado en la circulación lo constituia el cruce del canal Harvey, pues el puente levadizo construído para salvarlo debía servir para el tráfico rodado y el fluvial. Además, como tenía que dejar paso unas 1.400 veces al mes a la navegación, esto creaba un molesto retraso y estancamiento prolongado a la circulación rodada.

Para salvar este inconveniente, los ingenieros consultores Palmer and Baker Engineers, Inc., de Mobile, Alabama, y Nueva Orleáns, fueron encargados por las Instituciones oficiales locales del proyecto de un paso inferior del canal, que fuera capaz de mitigar el serio problema que este cruce venía creando. 
1 resultado de previos tanteos dió por enstrucción de un túnel bajo el canal Harvey. El túnel, recientemente terminado y puesto ya en servicio es de hormigón armado, cuyas secciones y dimensionado se pueden apreciar en los dibujos adjuntos. En realidad, está constituído por dos direcciones independientes, cada por dos direccionesta de dos bandas de circulación y separadas por medio de un muro central.

El túnel gemelo tiene aproximadamente $700 \mathrm{~m}$ de longitud total, pendiente del $5 \%$ descendiendo hacia el centro y un trozo de $330 \mathrm{~m}$ por debajo del canal. La calzada en el centro del canal se halla $11,30 \mathrm{~m}$ más baja que el nivel de aguas.

Para mejorar la capacidad de sustentación del terreno se hincaron 3.520 pilotes de madera. 0.500 toneladas de armaduras de acero $y$ unos $30.000 \mathrm{~m}^{3}$ de hormigón.

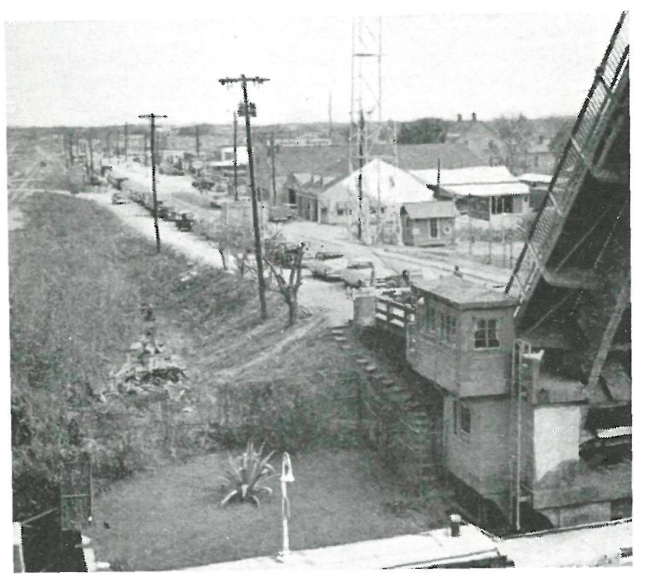

\section{Método de construcción}

El contratista de la obra propuso que el túnel se construyese a cielo abierto, pero en dos fases sucesivas para permitir la navegación fluvial durante el período de construcción. Para la construcción de la primera fase se preparó una ataguía que se internaba en el canal unos $70 \mathrm{~m}$, es decir, aproximadamente la mitad de la anchura del derinada esta primera fase se procedió a hacer otro tanto en la margen opuesta, desviando la navegación la margen opuesta, desviando la návegacion el desarrollo de estas dos fases no hubo necesidad de tapar el túnel.

E1 túnel se impermeabilizó con cuatro capas de protección o, si asi se quería, con revestido de gunita de $2,5 \mathrm{~cm}$ de espesor.

\section{Installaciones}

La circulación por el túnel se controla por medio de lámparas colocadas en las entradas y al empezar las rampas de acceso. De existir un contenido elevado de óxido de carbono, ser inundadas las calzadas o de producirse fuego en el túnel, estas lámparas darán un color rojo que anunciará peligro.

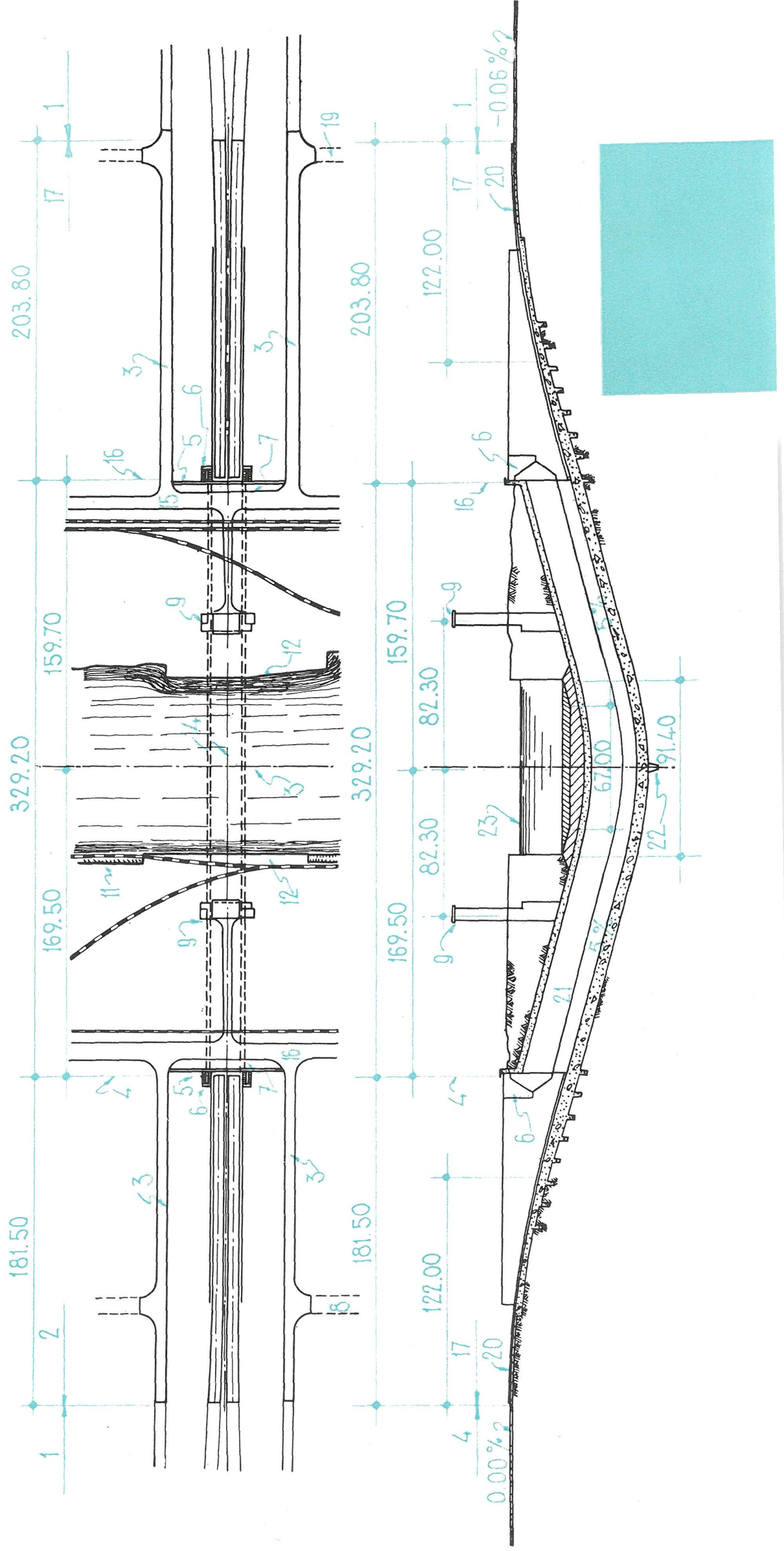


1 Construído por otros.

2 Límite del túnel.

3 Camino de servicio.

4 Portal del oeste.

5 Acera de 1,50 metros.

6 Escalera para peatones.

7 Bombas del portal.

8 Primera avenida.

9 Torre de ventilación.

10 Avenida Destrahan.

11 Muelle para madera.

12 Ataguía.

13 Canal Harvey.

14 Eje del túnel.

15 Avenida Peters.

16 Portal del este.

17 Límite del túnel.

18 Límite de la construcción de caminos de servicio.

19 Calle Greffer.

20 Bordillo.

21 Acera para peatones.

22 Bombas del centro.

23 0,00 nivel medio del agua.

24 Cota de la capa de arcilla.
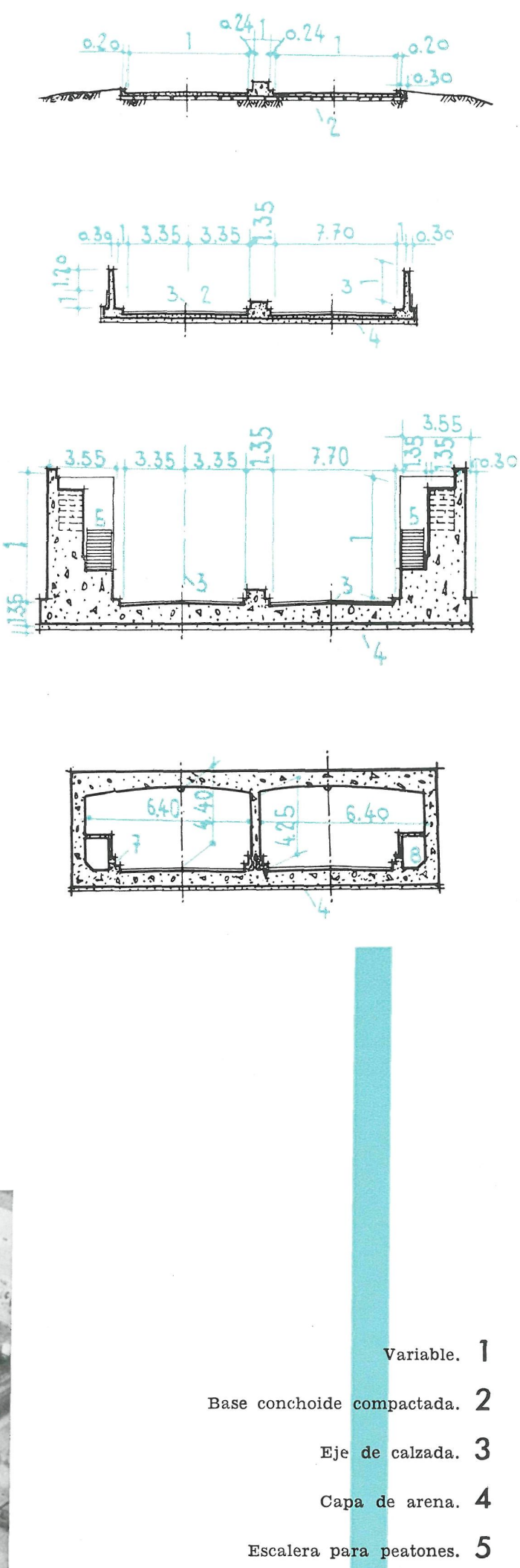

Escalera para peatones. 5

Cuatro membranas de impermeabilización 6 con $50 \mathrm{~mm}$ de mortero protector

o chapa de $6 \mathrm{~mm}$ y $25 \mathrm{~mm}$ de gunita.

$$
\begin{array}{r}
\text { Difusor de aire. } 7 \\
\text { Conducto para el aire. } 8
\end{array}
$$



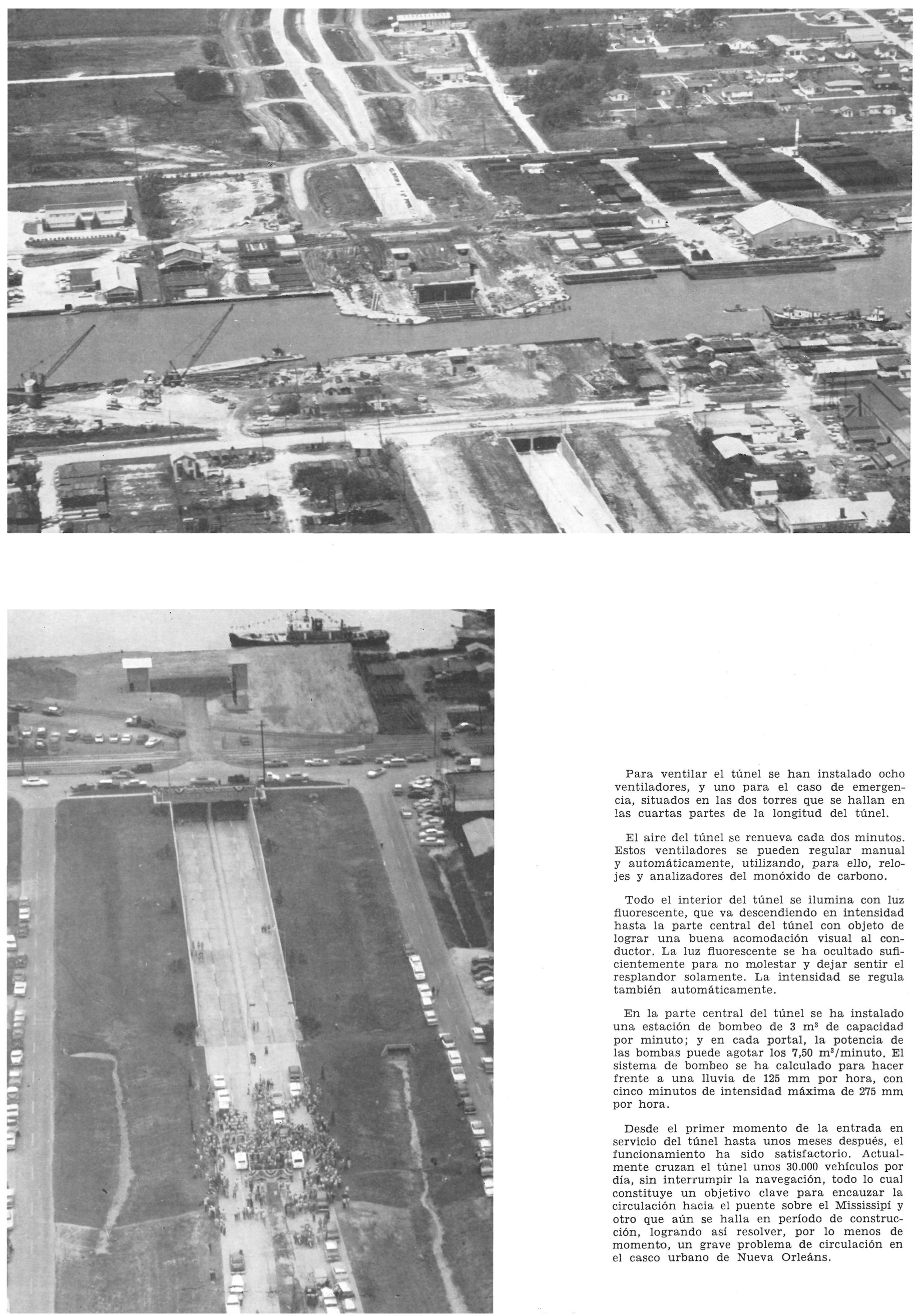

Para ventilar el túnel se han instalado ocho ventiladores, y uno para el caso de emergencia, situados en las dos torres que se hallan en las cuartas partes de la longitud del túnel.

El aire del túnel se renueva cada dos minutos. Estos ventiladores se pueden regular manual y automáticamente, utilizando, para ello, relojes y analizadores del monóxido de carbono.

Todo el interior del túnel se ilumina con luz fluorescente, que va descendiendo en intensidad hasta la parte central del túnel con objeto de lograr una buena acomodación visual al conductor. La luz fluorescente se ha ocultado suficientemente para no molestar y dejar sentir el resplandor solamente. La intensidad se regula también automáticamente.

En la parte central del túnel se ha instalado una estación de bombeo de $3 \mathrm{~m}^{3}$ de capacidad por minuto; y en cada portal, la potencia de las bombas puede agotar $\operatorname{los} 7,50 \mathrm{~m}^{3} /$ minuto. El sistema de bombeo se ha calculado para hacer frente a una lluvia de $125 \mathrm{~mm}$ por hora, con cinco minutos de intensidad máxima de $275 \mathrm{~mm}$ por hora.

Desde el primer momento de la entrada en servicio del túnel hasta unos meses después, el funcionamiento ha sido satisfactorio. Actualmente cruzan el túnel unos 30.000 vehículos por día, sin interrumpir la navegación, todo lo cual constituye un objetivo clave para encauzar la ción hacia el puente sob otro que aún se halla en periodo de construcción, logrando asi resolver, por lo menos de momento, un grave problema de circulación en el casco urbano de Nueva Orleáns. 